\title{
Spectrum Sensing Detection for Non-Stationary Primary User Signals Over Dynamic Threshold Energy Detection in Cognitive Radio System
}

\author{
Akil H. Wotaif ${ }^{1}$, Bashar J. Hamza ${ }^{2}$, Wasan Kadhim $\operatorname{Saad}^{3}$ \\ ${ }^{1}$ Engineering Technical College, Al-Furat Al-Awsat Technical University, Hilla, Babil, Iraq \\ mr.hassanalanezy181990@gmail.com \\ ${ }^{2}$ Engineering Technical College, Al-Furat Al-Awsat Technical University, Najif, Iraq \\ mrym_bashar@yahoo.com \\ ${ }^{3}$ Engineering Technical College, Al-Furat Al-Awsat Technical University, Najif, Iraq \\ was_basher@yahoo.com
}

\begin{abstract}
Nowadays, global evolution and technological revolution of wireless communication development, without observing the current radio spectrum assignment policy, cause the spectrum to be more depleted. Cognitive Radio (CR) is the most effective technique for solving the problem of spectrum scarcity, particularly in light of the desire to transmit data very quickly, as unauthorized users are able to use a spectral bandwidth. Whenever the owner of the license begins to use the domain, the connection of the unauthorized user will be cut until the authorized user ends his connection. Conventional methods have proven to be ineffective in detecting the frequency spectrum in comparison to dynamic Primary User (PU) methods, where the PU is either completely present or completely absent. In this paper, an algorithm is suggested which aims to improve energy sensing and increases the efficiency of detection through sensing and noticing the random dynamic move of the PU. This algorithm has been designed based on these rapid changes of the PU, and is called the Activity period $(A)$. In addition to activities of the PU during the sensing period, it also focuses on the dynamic threshold factor through which the detection is enhanced. The new formula is applied through the Dynamic Energy Detection-Dynamic Threshold (DED-DT) method with a constant false alarm rate analysed mathematically, realizing a reliable performance with a low signal-to-noise ratio (-12 dB) achieved through simulation. Therefore, the proposed algorithm showed relatively improved results when compared to those of traditional detection methods.
\end{abstract}

Keywords - Active period, Dynamic Energy Detection-Dynamic threshold, Low SNR, Non-stationary user.

\section{INTRODUCTION}

One of the main reasons for the spectrum congestion is the rapid growth in wireless communication, in addition to the large numbers of users. However, the spectrum lack is mostly due to the ineffective static spectrum allocations [1]. The traditional fixed distribution policy that defines the spectrum for authorized users has contributed to the scarcity of the spectrum, as shown in the Federal Communications Commission (FCC) report [2]. According to the survey of (FCC)'s Spectrum Policy task force in 2002, it is explained that there is a small utilization (from $15 \%$ to $85 \%$ ) of the frequency spectrum in a geographical region, time, and space. On the other hand, Cisco has contributed to a study on Global Mobile Data Traffic and showed that it will be a 7-fold increase in mobile data traffic from 2016-2021 [3].
More recently, Cognitive Radio (CR) has been used as an effective approach to enhance spectrum utilization through opportunistic spectrum sharing. The major concept of $\mathrm{CR}$ is sensing the unused frequencies in the spectrum, thus allowing the unlicensed users (secondary users) to utilize them without affecting the licensed users (primary users) [2].

Some researchers have shown that the spectrum is unlimited, but the problem is the poor management of this spectrum due to the fact that not the whole licensed spectrum is utilized; only a few parts are used, thus leading to the insufficient use of the spectrum [4]. To overcome all these problems, a smart wireless network called CR has emerged. Consequently, the primary 
importance of $\mathrm{CR}$ lies in inefficient spectrum utilization [4]. A CR can serve its users through the filling in the spectrum holes without causing harmful interference to the PU. This is achieved through continuous spectrum sensing to detect the PU. Once the licensed user is discovered, the CR should withdraw from the spectrum immediately to reduce the interference [5]. The four main functions of CR are: Spectrum sensing, Spectrum management, Spectrum sharing, and Spectrum mobility [6]. Spectrum sensing is one of the essential functions of CR which should be done first before allowing secondary users to reach a vacant licensed channel. In other words, it is a core of CR and includes the determination of the unused spectrum holes. Spectrum sensing includes obtaining the spectrum usage characteristics across multiple dimensions: time, frequency, space, and code, and identifying what type of signals are filling the spectrum [6] [7].

The interest of many previous studies and literature lies in studying the traditional fixed user as well as the fixed decision threshold.

In [8], the authors realized the approach closest to the reality represented in the activities and the process of traffic for the PU which moves randomly during the sensing period. The new parameter in this study is included within the detection algorithms, where these activities are confirmed by the actual user presence on the spectrum. Thus, the detection performance is enhanced and increases the performance of the sensor.

In [9], the authors focused on the use of spectrum as a major resource for both primary and secondary users through the use of prediction management technology and the grouping of $\mathrm{PU}$ activities according to the prediction process. In this study, the spectrum is reallocated and allowed to the secondary user at certain times.

In [10], the authors used the energy detection method to detect the CR spectrum. This study focused on the use of the probability of statistical properties to improve the accuracy of the false alarm probability, and then the estimated noise variance was used to obtain a suitable threshold for energy detection.

The proposed method significantly contributed to improving the detection performance and increases the efficiency of the system as explained in the results (section V). The method is closer to the real state where this algorithm is more flexible to deal with signal dynamics and PU dynamics at the same time. In other word, The main goal of this paper on the CR system is to demonstrate how the randomness of $\mathrm{PU}$ movement during sensor time affects the detection performance by deriving detection models related to the performance of the PU-A spectrum sensing.
Outline of the Paper

Section (II) involves the related work. Section (III) explains the spectrum sensing technique. Section (IV) shows the energy detection methodology. Section (V) explains the results and discussion. Finally, Section (VI) includes the conclusion.

\section{SPECTRUM SENSING TECHNIQUE}

Spectrum Sensing (SS) is the most crucial part of a CR system, being the most important function of this system. The secondary user should sense the spectrum efficiently[4]. Fast transmission opportunities are taken advantage of, so as to scan the spectrum whenever the PU is sensed to return to it. In other words, the main function of the SS system is to track and monitor the assigned spectrum continuously to make better use of these packages, and secondly, to prevent interference with primary (authorized) users so that all major parameters of PU users are predefined. If PU again wants to connect to the specified spectrum, SU should leave immediately without interfering with the PU signal. Hence, an increased spectrum efficiency will be ensured. Detecting active primary transmitters and receivers near CR devices is the most effective way to sense spectrum holes, however, there are some negative primary receivers such as televisions, some of which are difficult to detect in practice[1][11].

Due to the rapid expansion of the telecommunications system and its applications, the radio spectrum increases gradually being a natural and licensed resource, and it has been proven that the entire spectrum is not used in all places continuously. An idea behind the launch of a CR system is the fact that there are more systems and technologies geared towards wireless systems, and the demand for spectrum is expected to increase rapidly and significantly because of its attractiveness. The frequency spectrum is managed through standard agencies such as the Telecom Regulation Authority of India (TRAI) and the Federal Communications Commission (FCC) in the USA[1] [12].

There are three common techniques used for initial detection transmitters: energy detection method (ED), matched filtering method (MF), and the Cyclostationary Feature Detection (CFD) method [13] [14].

For spectrum sensing, there are two important probabilities assumed: the first is the probability of detecting and determining, with the $\mathrm{H} 1$ hypothesis, which indicates the possibility of the algorithm correctly revealing the presence of the PU, being a valid decision; the second is the possibility of false alarm and determining, assuming $\mathrm{HO}$, which indicates the algorithm making an incorrect declaration concerning the existence of the primary signal, thus being a false decision. It is therefore considered a must for the detection rate be somewhat high if the 
algorithm works well with the possibility of a false alert being low [15].

\section{ENERGY DETECTION METHODOLOGY}

When information about PU (PU) is unknown in CR, the PU detection is a commonly used method of detecting energy (sometimes called radiometric). Energy Detection (ED) can be implemented in both frequency and time domain [16].

It will be particularly suitable for spectrum sensing broadband services when the registry cannot collect enough information about the PU signal. In detecting a signal, the signal is either present or absent. The present is characterized by two hypotheses, the first one is known as an alternate hypothesis, and the second one is known as a null hypothesis . Finally, the signal the secondary user receives will be compared to a predetermined threshold to determine whether or not the PU is present [3] [12].

To clarify, when the threshold is unnecessarily high, it is most likely that there is a positive correlation between the number of errors and the probability of false alarm: the more errors are detected which may cause active interference to the PU, the higher the probability of a false alarm to occur, eventually resulting in a decrease in Spectrum use. On the other hand, if the specified threshold is low, then the probability of a false alarm increases, thus leading to an increase in Spectrum use. Therefore, the exchange can be considered accurate when determining the energy threshold, in case it targets the possibility to reuse the spectrum from the unused spectrum and false alarm [3].

Among the advantages of the energy detection method is the fact that prior information about PUs is not required, as computational implementation and complexity are generally low. As well, a relatively short time of monitoring is required. Although the energy method has a low complexity, it does have its drawbacks. One downside is that detection performance is poor under low signal-tonoise ratio scenarios as an asymmetric detection model; another is its inability to distinguish between a PU signal and interference from other transmitters. Consequently, adaptive signal processing, such as interference suppression, cannot be used. Moreover, uncertainty in the noise level may lead to a further deterioration in performance, which can be overcome by in [1].

Because of the simplicity of application and low complexity calculations, this method is often considered to be the most common method in a CR system, and is referred to as the blind method. The fact that this technology (ED) depends on the strength of the noise signal (or energy) and ignores the type and characteristics of the signal is a positive aspect, yet the main problem is that it suffers from impotence: among other defects, the time sensor cannot distinguish the highest time the PU signal Cognitive signal performance degrades in the event of noise uncertainty [12].

The Energy signal is calculated by the following Equation: $\mathrm{E}=\sum_{n=1}^{L-1}|X(n)|^{2}$

Where $\mathrm{E}$ represent the energy value, $\mathrm{L}$ is the number of sample, and $\mathrm{X}(\mathrm{n})$ is the received signals at $\mathrm{CR}$ nodes

In the next part of this paper, a mathematical description will be presented to define detection parameters and facilitate handling this task, providing a mathematical analysis as is shown below.

\section{A) PU Non-Stationary Concept}

The concept of CR mainly relies on dynamic access to the spectrum because it allows unauthorized users to use the spectrum owned by licensed PUs as long as it interferes at the minimum with the PU.

It is known that the crucial thing for $\mathrm{CR}$ is spectrum sensing, and the sensing procedure should be to establish a cycle named to a Non-stationary user or dynamic PU [10]. This description has been done due to activities and events that he performs during the sensing period $(A)$.

Studies have acknowledged this to be more general and realistic for practical applications, as in practical CR, it is desired for the PU dynamic that the sensing period is shorter than the transmission period, forming a sensing cycle to allow more time secondary users to transmit data [4].

Concerning the configuration of the sensing cycle that has two parts (sensing and transmission interval), the shorter the sensing period is, the higher user productivity and more transmission time there will be [4]. The secondary user must protect both the PU and its productivity.

One common way to help discover the spectrum is the random movement of basic user activity. This movement means changing the PU status from inactive to busy or vice versa during the sensing period. This paper has focused on patterns of random change for the PU throughout the sensing period.

Also, in this method, the focus was on the parameter of radio signal dynamics and minimizing the effect of noise. The main problem with the low performance of the energy detection method in the $\mathrm{CR}$ system is due to the uncertainty in the noise factor, which will lead to unauthorized secondary access to the main user network and its dangerous consequences. In other words, the higher the noise factor is, the greater the direct negative impact on the detection performance will be, and thus the efficiency of this system deteriorates. Therefore, this factor is considered to be one of the important parameters in the sensor process [17] [13], which can be represented as such through the following distribution: 
through the following distribution: $\sigma^{2} \varepsilon\left[p \sigma_{n}^{2}, \sigma_{n}^{2} / p\right]$

Where $\sigma_{n}^{2}$ is the variance of noise and $\mathrm{p}$ is noise uncertainty factor (NU), which is close to 1 (i.e. $\mathrm{p} \geq 1$ ).

To improve sensing with high reliability, this is applied according to the hypothesis of the double dynamic threshold indicating dynamic detection theory with the assumption that the false alarm rate is constant, $d$ is close to $1(d \geq 1)$ and the dynamic term for $\left\{\lambda_{D} \varepsilon\lfloor d \lambda, \lambda / d\rfloor\right.$.

The effects of the change in dynamic threshold factor (d) are taken in this study, regardless of the presence or absence of the noise instability factor, assuming that the noise uncertainty factor is constant and is equal to one. It focused on studying the double dynamic threshold, and how it improved the likelihood of detection and its results. Regardless of the presence of uncertainty of noise, the use of double dynamic leads to much better results than the use of a single static threshold or a dynamic threshold [13].

\section{B) The Traditional Energy Detection over CFAR-Fixed Threshold Detection}

In a fixed spectrum sensing, it is estimated that the PU is static, also its unchanged activity remains, i.e. the PU is either totally existing or does not exist at all within the sensing duration. Therefore, the absence or presence of the PU signal can be determined based on some assumptions and the observed signal model $\mathrm{X}(\mathrm{n})$ as in Equation (2) below [18]. It is noticed here that the signal observed according to hypothesis $H 1$ includes PU signal $\mathrm{s}(\mathrm{n})$, whereas the noise $\mathrm{w}(\mathrm{n})$ is only to be detected if corrupted by noise for the observed signal under $H O[14]$.

Constant False Alarm Rate (CFAR) technology is one of the techniques used to reduce the effect of noise in energy detections, where the maximum values of test statistics and different sequences are determined and the best threshold is selected to result in good detection and a low rate of false alarm, which is significant for the Spectrum sensing results.

The Spectrum sensing rule is a Binary Hypothesis for testing a problem. The received signal composition at the secondary user under this binary hypothesis is defined for nth sample, $\quad 1 \leq n \leq L$, as follows [12]:

$$
\begin{array}{ll}
X(n)=\{w(n) & H_{0}(\text { vacant }) \\
X(n)=\{w(n)+s(n) & H_{1}(\text { occupied })
\end{array}
$$

Where $\mathrm{n}=1,2.3 .4, \ldots \ldots \mathrm{L}$, represents the number of samples, and $X(n), w(n)$ and $s(\mathrm{n})$ are the received signals at
CR nodes, white noise samples, and transmitted signals at PUs nodes respectively [6][14].

The decision of the energy detection is the test of the following hypothesis. Test statistics for the energy detection method can be obtained through the following [9]:

$$
D(X)=\frac{1}{L} \sum_{n=1}^{L-1}|X(n)|^{2}
$$

Here, $L$ is the number of samples taken in the observation period, and $\mathrm{D}(\mathrm{X})$ is the test statistics. To obtain a single threshold value $\boldsymbol{\lambda}$, the presence or absence of a licensed station can be declared as:

$D(X) \geq \lambda, H_{1}$ : licence termal is present

$D(X) \leq \lambda, H_{1}$ : licence termal is absent

The noise variance is considered to be fixed if the noise uncertainty $(N U)$ is ignored.

$$
\begin{aligned}
& D(X) \sim\left\{\operatorname{Normal}\left(\sigma_{n}^{2}, \frac{2}{L} \sigma_{n}^{4}\right)\right. \\
& D(X) \sim\left\{\operatorname{Normal}\left(\sigma_{n}^{2}+\sigma_{s}^{2}\right), \frac{2}{L}\left(\sigma_{n}^{2}+\sigma_{s}^{2}\right)^{2}\right. \\
& H_{0}(\text { vacant }) \\
& H_{1}(\text { occupied })
\end{aligned}
$$

Where $\sigma_{n}^{2}$ and $\sigma_{s}^{2}$ are the variance of noise and the variance of primary signal respectively. From the two hypotheses above, the probability of false detection $p f$, probability of detection $p d$ and the probability of misdetection $\mathrm{pm}$ can be extracted as

$$
\begin{aligned}
& p d=\operatorname{pro}\left(D(X) \geq \lambda / H_{1}\right)=Q\left(\frac{\lambda-\left(\sigma_{n}^{2}+\sigma_{s}^{2}\right)}{\sqrt{2 / L}\left(\sigma_{n}^{2}+\sigma_{s}^{2}\right)}\right) \\
& p m=1-p d=1-Q\left(\frac{\lambda-\left(\sigma_{n}^{2}+\sigma_{s}^{2}\right)}{\sqrt{2 / L}\left(\sigma_{n}^{2}+\sigma_{s}^{2}\right)}\right) \\
& p f=\operatorname{pro}\left(D(X) \geq \lambda / H_{0}\right)=Q\left(\frac{\lambda-\sigma_{n}^{2}}{\sqrt{2 / L} \sigma_{n}^{2}}\right)
\end{aligned}
$$

Where $\mathrm{Q}(\cdot)$ indicates the standard complementary Gaussian distribution function $(\mathrm{CDF}), \lambda$ represents the threshold value. $P d, P m$, and $P f$ represent detection probability, missing probability, and false alarm probability respectively, and are linked to the relationship between the probability of erroneous discovery, the number of samples observed, and the noise factor during the sensing period. The mathematically constant threshold can be found from in (7) and replaced into the probability of detection in(4), which is a major parameter in determining the PU signal detection ratio. 
$\lambda=Q^{-1}(p f) \times \sqrt{\frac{2}{L}} \sigma_{n}^{2}+\sigma_{n}^{2}$

Where $\lambda$ represents fixed threshold.

For the detection equation $(p d)$, it mainly depends on the threshold $[\lambda]$ value ,by Applying (7) into (4). This is achieved through the equation (8) and derivation below:

$p d=Q^{-1}\left(\frac{Q^{-1}(p f) \times \sqrt{\frac{2}{L}} \sigma_{n}^{2}+\sigma_{n}^{2}-\left(\sigma_{n}^{2}+\sigma_{s}^{2}\right)}{\sqrt{\frac{2}{L}}\left(\sigma_{n}^{2}+\sigma_{s}^{2}\right)}\right)$

$Q^{-1}(p d) \times \sqrt{\frac{2}{L}}\left(\sigma_{n}^{2}+\sigma_{s}^{2}\right)=Q^{-1}(p f) \times \sqrt{\frac{2}{L}} \sigma_{n}^{2}+\sigma_{n}^{2}-\left(\sigma_{n}^{2}+\sigma_{s}^{2}\right)$

$Q^{-1}(p d) \times \sqrt{\frac{2}{L}}\left(\sigma_{n}^{2}+\sigma_{s}^{2}\right)=Q^{-1}(p f) \times \sqrt{\frac{2}{L}} \sigma_{n}^{2}-\sigma_{s}^{2}$

$\sigma_{s}^{2}=Q^{-1}(p f) \times \sqrt{\frac{2}{L}} \sigma_{n}^{2} Q^{-1}-(p d) \times \sqrt{\frac{2}{L}}\left(\sigma_{n}^{2}+\sigma_{s}^{2}\right)$

$\gamma=\frac{\sigma_{s}^{2}}{\sigma_{n}^{2}}=S N R$

where $\gamma$ is the signal to noise ratio.

$S N R=Q^{-1}(p f) \times \sqrt{\frac{2}{L}}-Q^{-1}(p d) \times \sqrt{\frac{2}{L}}(1+S N R)$

$S N R^{2}=\frac{2}{L}\left[Q^{-1}(p f)-Q^{-1}(p d) \times(1+S N R)\right]^{2}$

$L=\frac{2 \times\left[Q^{-1}(p f)-Q^{-1}(p d) \times(1+S N R)\right]^{2}}{[S N R]^{2}}$

Where $L$ represents the number of samples[11].

In (9), the $L$ is associated by the desired PD at $\mathrm{SNR}$ value for a particularized pf system that is based on the constant false alarm rate principle (CFAR), i.e. the pf can be set to a determined value to select a threshold $(\lambda)$ value and obtain the desired probability of detection (PD).

\section{C) The Proposed Dynamic Energy Detection Non- stationary Primary User under CFAR-Double Dynamic Threshold Detection}

Conventional spectrum sensing for $\mathrm{CR}$ presumes the $\mathrm{PU}$ is stable or stationary within the sensing period and remains inactive or in a fixed status, whereas the primary signal may be unstable or non-stationary and changes states with a sensing period. Several studies have accepted and approved this fact, and utilized the activity modality of the PU to enhance the above-mentioned method in terms of performance, such as spectrum management and spectrum sensing whenever the PU is non-stationary and present during the spectrum period with Activity period A. This paper takes into account the scenario of random activities of the basic user movement during the sensing period and is called the activity period $(A)$, where $0<A<1$. In this case, the traditional methods cannot be applied because the PU hesitates randomly during the observed period.

The performance detection of $p_{D D / A}$ represents the activity period $(A)$ for energy detection when there are no activities for this user $A=0$, regardless of the SNR values indicating that the user is absent and that the received signal is noise. On the other hand, $A=1$ indicates that the user is completely present in this case, and increases the discovery rate significantly. It is therefore noted that the $p_{D D / A}$ performance is primarily associated with dynamic factor acquisition and activity of the PU from both operators for improved detection.

Through the rest of the remaining notice results, $X_{A E D}$ is the observed signal which consists of two parts including PU signals corrupted by noise with total power $\sigma_{n}^{2}(\gamma+1)$ of length $L A$, including PU signals corrupted by noise with total power $\sigma_{n}^{2}$ of length $L A$, and another part including only noise with power $\sigma_{n}^{2}$ of length $L(1-A)$, whereas the normal distribution with zero mean and variance is $\sigma_{n}^{2}(\gamma \cdot A+1)$. The static $X(n)$ test is used for the result of the sum of the energy of approximately two different parts. Whenever it is related to a Gaussian distribution, the Energy detection calculates the Energy of the observed signal, thus the PU signal location will affect the test output with the following distribution [16].

$$
X_{A E D} \sim N\left((\gamma A+1) \sigma_{n}^{2}, \frac{2}{L}(\gamma A+1)^{2} \sigma_{n}^{4}\right)
$$

Where $N$ is Normal distribution, $\gamma$ is the signal to noise ratio (SNR) at the secondary user, $\sigma_{n}^{2}$ is Noise variance, $\boldsymbol{L}$ is number of samples and $\boldsymbol{A}$ is Activity period. According to the effect of the PU signal activity period $D A(X)$, statistics can be performed by calculating the cumulative distribution function through AWGN channel in (3) [9].

$$
X(n)_{A E D}=\sum_{n=0}^{L A-1}|s(n)+w(n)|^{2}+\sum_{n=L A}^{L-1}|w(n)|^{2}
$$

Where $\mathrm{w}(\mathrm{n}), \mathrm{s}(\mathrm{n})$ and $X(n)_{A E D}$ denote additive white Gaussian noise with zero mean and variance $\sigma^{2}$, the transmitted signal from $\mathrm{PU}$, and the received signal at $\mathrm{CR}$ (i.e. secondary user) with Activity period respectively.

To calculate the statistical test $D_{A}(X)$, L samples are used for the observed signal, and the PU signal location does not affect the statistical distribution of the test. In the conventional energy detection algorithm, performance is 


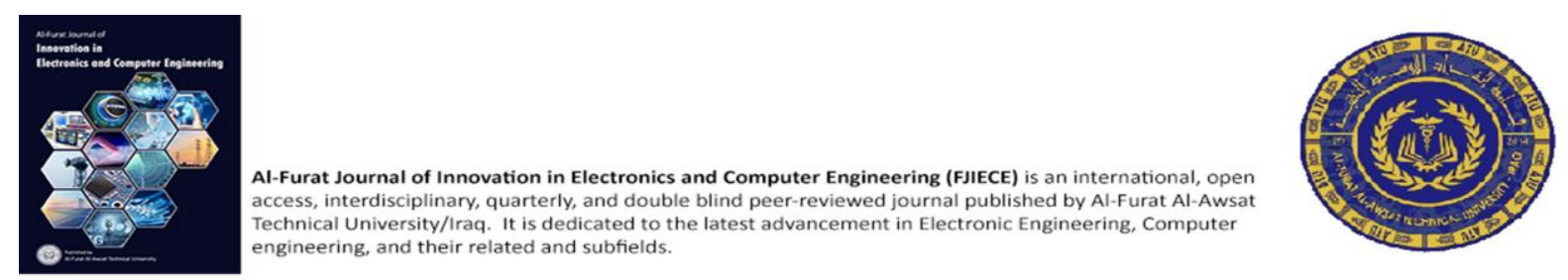

lower with uncertainty about noise being a fixed threshold, which indicates that the choice of fixed threshold is no longer good and appropriate, especially when being uncertain about noise, so a flexible dynamic threshold is needed instead of when necessary [6]. The typical approach used to formulate the PU-A decision threshold in this paper is a signal-based CFAR approach. In comprehensive CFAR, a principle is adopted in the sense that the false alarm rate probability is fixed to a small value so that the detection probability of the maximum is increased. The dynamic threshold $D T$ is calculated as a variable function, and the threshold is affected according to fluctuations and conditions that occur during the signal path with the assumption that the false alarm probability and the variable and affected detection principle are established according to those, having the same fluctuations and conditions that fully targeted the threshold. These are given as follows:

$$
\lambda_{d}=\frac{\sigma_{n}^{2}}{d}\left(\sqrt{\frac{2}{L}}\left(Q^{-1}(p f)+1\right)\right.
$$

Where $\lambda_{d}$ represents dynamic threshold.

Whenever a PU signal with an Activity period $(A)$ is detected, the $\mathrm{A}$ is introduced into the distribution for the hypotheses $H_{1}$ only for the Dynamic Energy Detection (DED). Consequently, the probability of detection performance can be determined by the proposed updated formulas, given the effect of the random movement of the PU-A, which is referred to as A. The activity period is in a closed-form, compared to the test of the cumulative distribution function which was formulated for the fixed PU who does not realize the random movement A that may occur during the sensing period.

The deterministic effects of fading in the AWGN channel are not taken into consideration, and therefore a Dynamic Energy Detection chart is presented to demonstrate the decision regarding the presence of the signal $s(n)$ as in Hypothesis (Equation2). This section is presented a form of the dynamic spectrum sensing formula with the dynamic threshold DED-DTD under CFAR and compares with the models conventional over AWGN channel, which is a good and ideal example of comparing the different methods of sensing the spectrum in CR networks. the $D E D-D T D$ method is assumed due to fluctuations in signal level variation that is to the change in the noise level, reducing the sensitivity of the sensing and eventually decreasing in the detection accuracy in CR networks and resulting in harmful interference with the $\mathrm{PU}$, as the traditional energy detection algorithm which depends on a fixed threshold which is very sensitive to noise fluctuations. Given that the fixed threshold is no longer valid at the stability of the noise level, it is therefore important to suggest a dynamic threshold DT scenario and an effective approach to address noise fluctuations as well as to enhance detection sensitivity.
Therefore, the new $D E D-D T$ proposal based on the DT scenario Dynamic predisposition will be taking into consideration the random return of the PU during the sensing process $\left(p_{D D / A}\right)$, which can be calculated in a closed model on the AWGN channel by the cumulative distribution function of statistics [8] [10]. The test in (3) is applied with the compatibility threshold calculated in(Equation 12). Accordingly, the detection performance is evaluated when the primary signal that shows the Activity period $(A)$ is detected by applying $\lambda_{D}$ into $X_{A E D}$, so as to get:

$$
P_{D D / A}=Q\left(\frac{\frac{1}{d}\left(\sqrt{\frac{2}{L}}\left(Q^{-1}(p f) \sigma_{n}^{2}+\sigma_{n}^{2}\right)\right)-d(S N R \times A+1)}{\sqrt{\frac{2}{L}} d(S N R \times A+1)}\right)
$$

Where $\quad p_{D D / A}$ is the approximate equation for the probability of performing a closed-form detection of a $D E D$ scheme based on the dynamic threshold dT, which has been tested over the AWGN channel when viewing the

PU and $A$ represents the activity period. $p_{D D / A}$ can be used to analyze both false alarm and detection probabilities under $H_{0}$ and $H_{1}$ respectively, assuming the dynamic threshold coefficient $(d \geq 1)$ and specifying a range of $\mathrm{dT}$ $[\lambda / d, d \lambda]$ values. As for the special cases when $\mathrm{d}=1$ and $\mathrm{d}=0, p_{D D} / \mathrm{A}$ is the conventional detection probability (PU signal existent) and is equivalent to the traditional false probability rate (PU signal not existent). As well, the SNR required to achieve $p d \geq p_{D D / A}$ can be obtained as follows:

$$
S N R=\frac{Q^{-1}(p f)-Q^{-1}(p d)}{Q^{-1}(p d)-\sqrt{\frac{2}{L}}}
$$

The equation represents the signal-to-noise ratio (SNR) with the change in probability of the detection ratio and the number of samples observed when assuming that the false alarm rate is constant. The improvement of the detection, which has been explained above achieved by applying equation 13 which represent the main model of this work. It is worth noting that the traditional detection uses the fixed threshold $(\lambda)$ (Equation 7 ) with the probability of detection equation (pd) (Equation 4) while the dynamic detection used the dynamic threshold coefficient (d) (the single dynamic threshold has been called in this paper due to the effect of (d) is on the probability of false (pf) only (Equation 7). This work took into account the effect of (d) on the probability of false (pf) (equation 6) and the probability of detection (pd) (equation 4) with a dynamic 


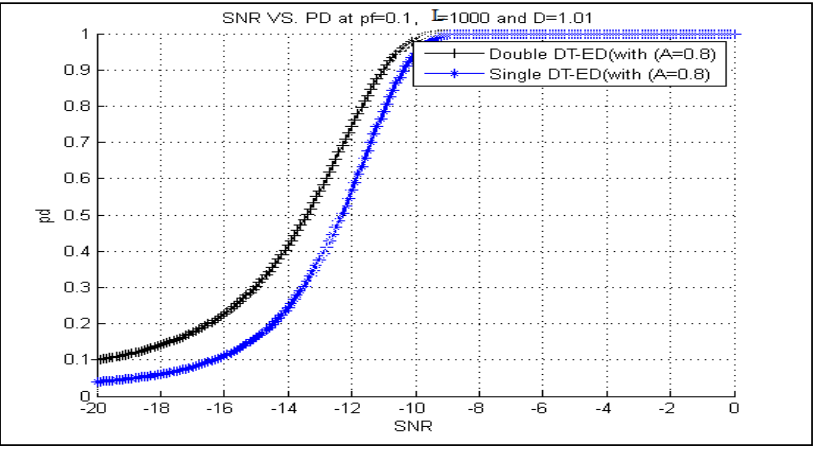

Figure 5: Probability of Detection(PD) vs. SNR

$\mathrm{pf}=0.1, \mathrm{~L}=1000, \mathrm{~d}=1.01$, No. of iterations $=100$ and $\mathrm{A}=0.8$

This figure includes the probability that the PU (i.e. primary partially present) would occur through determining the value of the Active period $(A)$ or the percentage of the PU's presence, as well as by simulating the algorithm on the AWGN channel. It has been shown that the higher this percentage is, the more effective the discovery percentage will be (unlike the conventional discovery which simply assumes whether the above model was more efficient), and gives channel usage a real look reducing the overlap rate as it is ready to deal with user licenses again. Finally, in all the charts shown above, the simulation experiment demonstrated that using the dynamic double threshold produces the best results from using the single threshold with simulations.

Figure (6) shows the probability of detection (PD) against the dynamical threshold, when the value of constant false alarm rate $\mathrm{pf}=0.1$, while the number of samples, in this case, is different. We may notice that the detection increases as the dynamic factor increase. The proposed method achieved a good achievement in light of the reduced number of assumed samples with a low signal-tonoise ratio.

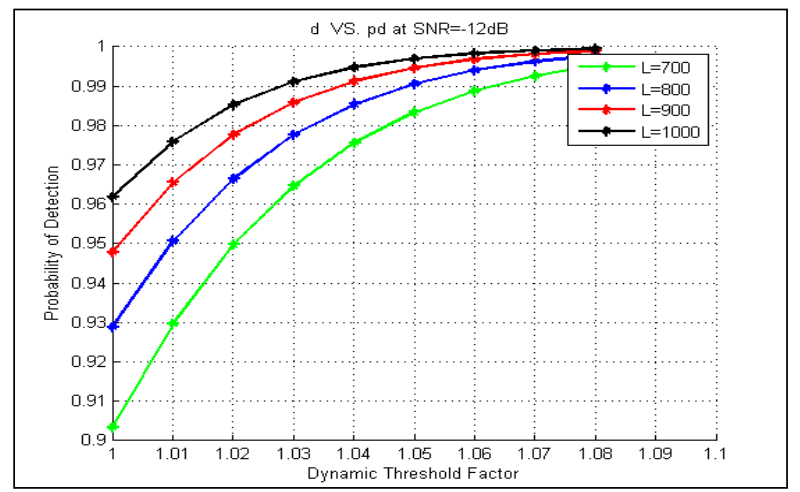

Figure 6: Probability of Detection(PD) vs. Dynamic threshold with, No. of iterations $=100$ and $\mathrm{pf}=0.1$

The proposed method results for PU signal detection are better than all the other methods explained in this paper as shown in the figures above. this is achieved by applying the equation 13 .

\section{CONCLUSION}

This paper introduced a method for sensing the spectrum through sensing energy, which mainly depends on the dynamic threshold method. It also shed light on the activities of the primary user, which take place during the so-called activity period (A) within the sensing period, where the primary user makes random traffic during both the sensing and transmission period. This scenario is the realistic approach for the primary user who has freedom of communication to use its dedicated channels in the spectrum, so the dynamics of the threshold, as well as the random movement and their interaction with the energy detector are studied at the same time, showing that the traffic activities of the primary user and the percentage of discovery increase correlatively. As well, a slight change in the value of the dynamic threshold occurs, which is offset by a noticeable improvement in the performance of the detector. The results show a remarkable improvement in the performance of the detector compared to the fixed detection method and the traditional fixed threshold. It is therefore concluded that the dynamic user and the dynamic threshold are the main parameters that caused the reliability of the detector and the performance of the detector. These proposed formulas can contribute significantly to enhancing the use of the unused spectrum.

\section{REFERENCES}

[1] A. Sciences, M. Scientific, and P. Corp, "Research Article A New Adaptive Two Stage Spectrum Sensing Technique in CR System for Different Modulation Schemes $\mathrm{Dr}$. Hadi $\mathrm{T}$. Ziboon and 2 Ahmed A . Thabit Department of Communications Computers, AL-Rafidain University / College , Baghdad , Iraq,” vol. 13, no. 11, pp. 856-863, 2016.

[2] N. Goyal, S. Mathur, C. Engineering, and U. S. Nagar, "Spectrum Sensing and Energy Efficiency Strategies in CR NetworksPerspective and Prospects," vol. 13, no. 5, pp. 2395-2411, 2018.

[3] P. K. Verma, "Study and Analysis of CR FOR," no. January 2019.

[4] P. Sharma, C. E. Branch, and C. E. Branch, "Dynamic Energy Detection Approach for Spectrum Sensing in Cognitive Radio Networks," vol. 1, no. 4, pp. 1-6, 2012.

[5] C. Korumilli, C. Gadde, and I. Hemalatha, "Performance Analysis of Energy Detection Algorithm in Cognitive Radio," vol. 2, no. 4, pp. 1004-1009, 2012.

[6] M. Al-rawi, "Review on the Performance of Non-Cooperative Spectrum Sensing Based on Energy Detection,” vol. 5, no. 5, 2017.

[7] G. Mahendru, "Effect of Dynamic Threshold on Sensing Duration for Robust Detection in CR Systems for Low SNR Scenarios," pp. $0-4,2017$.

[8] K. Chang, S. Member, and B. Senadji, "Spectrum Sensing Optimisation for Dynamic PU Signal," vol. 60, pp. 3632-3640, 2012

[9] L. Csurgai-horváth and J. Bitó, "Primary and secondary user activity models for cognitive wireless network," no. May, 2017.

[10] L. Xiao, H. Pin-Han, and P. Limei, "Statistical Properties of Energy Detection for Spectrum Sensing by Using Estimated Noise Variance," 2019. 
